\title{
Lasing and Mode Switching in Circular Bragg Nanoresonators
}

\author{
Jacob Scheuer \\ Center for the Physics of Information, California Institute of Technology, MC 128-95, 1200 E. California Blvd., Pasadena, CA 91125, USA \\ koby@caltech.edu \\ William M. J. Green, Guy DeRose and Amnon Yariv \\ Department of Electrical Engineering, California Institute of Technology, MC 128-95, 1200 E. California Blvd., Pasadena, CA 91125, USA
}

\begin{abstract}
We demonstrate low-threshold lasing at telecommunications wavelengths from high quality circular semiconductor nanoresonators employing radial Bragg reflector. Single-mode emission and mode switching are observed at room temperature under optical pumping. (C2005 Optical Society of America

OCIS codes: 230.5750 Resonators; 350.3950 micro-optics
\end{abstract}

\section{Introduction}

Circular resonators are key ingredients in the realization of many basic components needed for advanced optical communication systems [1]. During the last decade, numerous circular-resonator based applications such as filters [1], add/drop multiplexers [2], modulators [3], and delay lines [4] have been suggested and demonstrated. The applicability of circular resonators was shown to extend beyond telecommunication to the fields of sensing [5], spectroscopy, and standardization [6], as well as to basic research in QED, nonlinear optics, and other related fields [7]. For many of these applications, the resonator is required to exhibit low losses (high $Q$ ), and small modal volume. For conventional resonators, which utilize total internal reflection (TIR) as the radial confinement mechanism, these requirements are mutually contradictory.

Here, we demonstrate circular nano-lasers employing Bragg reflectors as the radial confinement mechanism. The reflector design is based on a novel radial coupled mode theory [8], yielding chirped gratings, and thus, enabling efficient confinement of the electromagnetic field in sub-micron dimensions.

\section{Design concept and theory}

Figure 1 shows an SEM micrograph of semiconductor nano-lasers designed for angular propagation coefficients of $m=0$ (a) and $m=4$ (b). The internal disk serves as the cavity while the concentric rings are the radial reflector, both designed to efficiently confine a specific mode in the cavity. The cavity radius is approximately $150 \mathrm{~nm}$ the $m=0$ resonator, making it the smallest demonstrated semiconductor laser in telecommunication wavelengths. The radius of the $m=5$ resonator is $560 \mathrm{~nm}$. For a circular cavity to support a radial mode with an angular propagation coefficient of $m$, the radius of the inner disk, $\rho_{0}$, and the grating profile $\Delta \varepsilon$ must satisfy [8]:

$$
\Delta \varepsilon(\rho)=\left\{\begin{array}{cc}
-2|\alpha| & \sin \left\{\text { phase }\left(H_{m}^{(1)}\left[k_{0} n_{e f f} \rho\right]\right)\right\}<0 \\
0 & \sin \left\{\text { phase }\left(H_{m}^{(1)}\left[k_{0} n_{e f f} \rho\right]\right)\right\} \geq 0
\end{array} \quad J_{m}\left(k_{0} n_{e f f} \rho_{0}\right)=0\right.
$$

where $k_{0}, n_{\text {eff }}$ and $\alpha$ are respectively the desired resonance wavenumber, the slab effective index and the perturbation strength. $H_{m}$ and $J_{m}$ are respectively the Hankel and the Bessel functions of order $m$. The widths of the resulting layers are determined by the zeros and extrema of the Bessel function of order $m$. Due to the circular geometry, the optimal layer widths required to confine the light in the central pillar are not constant [9, 10] but rather monotonically decreasing with the radial distance. The chirped nature of the gratings, which can be clearly seen in Fig. 1(a), is highly important. Deviation from that profile will result in a non-optimal phase relations between the partially reflected waves from the Bragg layers and in a weaker confinement of the electromagnetic field. Actually, the grating profile (1) is of "second Bragg order" in the sense that the second term in the Fourier expansion of (1) is the term responsible to the radial reflection. Such an approach induces efficient vertical emission from the device which decreases the overall $Q$ on one hand but enables simple observation of the intensity pattern evolving in the device on the other. The condition on the pillar radius can be intuitively understood The modal field profile of the device is given by a Bessel function of order $m$ in the central pillar and an exponentially decaying Bessel function in the grating area where the decay constant, $k$, is determined by the perturbation strength $\alpha$ according to $k=\alpha / 2 n_{0}^{2}$ 


\section{QME1}

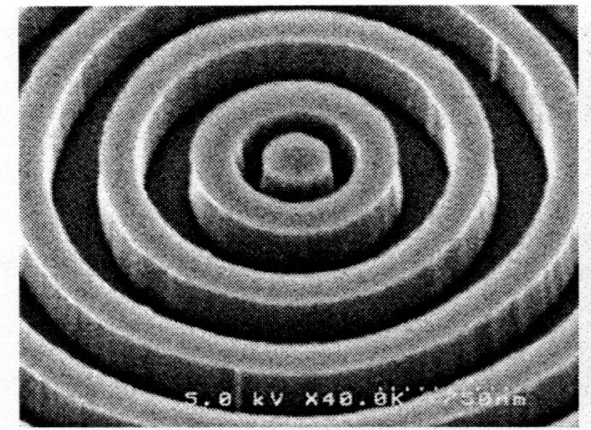

(a)

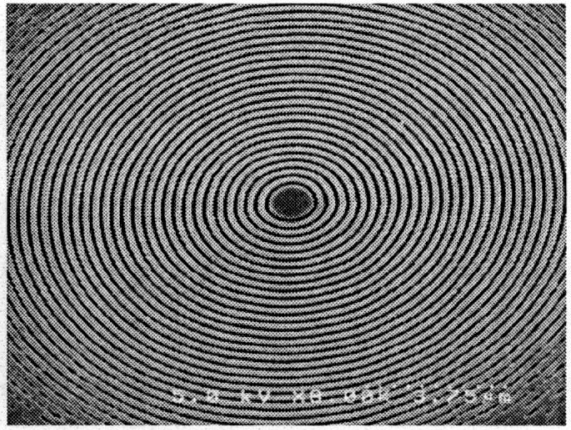

(b)

Fig. 1. Circular nanoresonators for (a) $m=0$ and (b) $m=4$.

\section{Fabrication and measurement}

Nano-cavities of several geometries were fabricated within a $250 \mathrm{~nm}$ thick membrane of InGaAsP with six $75 \AA$ quantum wells $(\mathrm{QW})$ positioned at the center. After the $A B R$ patterns were etched into the active material, the original InP substrate was removed and the membrane was transferred to a sapphire plate using an ultraviolet curable optical adhesive to improve the vertical confinement of the electromagnetic field in the device [11, 12]. The effective index of the membrane was found to be approximately 2.8 for the $H_{z}$ polarization and 2.09 for the $E_{z}$ polarization. Since the $H_{z}$ polarization is more confined than the $E_{z}$ polarization and the optical gain of the compressively strained QW structure used favors the $H_{z}$ polarization [13], we optimized the radial structure to this polarization.

The near-field (NF) intensity pattern and the emitted spectrum of the lasers were examined at room temperature under pulsed optical pumping. The $890 \mathrm{~nm}$ pump beam from a Ti:Sapphire mode locked laser was focused through the transparent sapphire substrate on the backside of the sample with a 50X objective lens. The position of this lens was used to control the size and the position of the pump spot. $50 \%$ of the pump beam intensity was split by a $3 \mathrm{~dB}$ beam-splitter and was focused on a broad area detector to obtain the pump power. A 20X objective lens was used to collect the vertical emission from the front side of the sample and to focus it on an IR camera to obtain the NF intensity pattern and to couple the light into a multi-mode fiber to obtain the emitted spectrum.

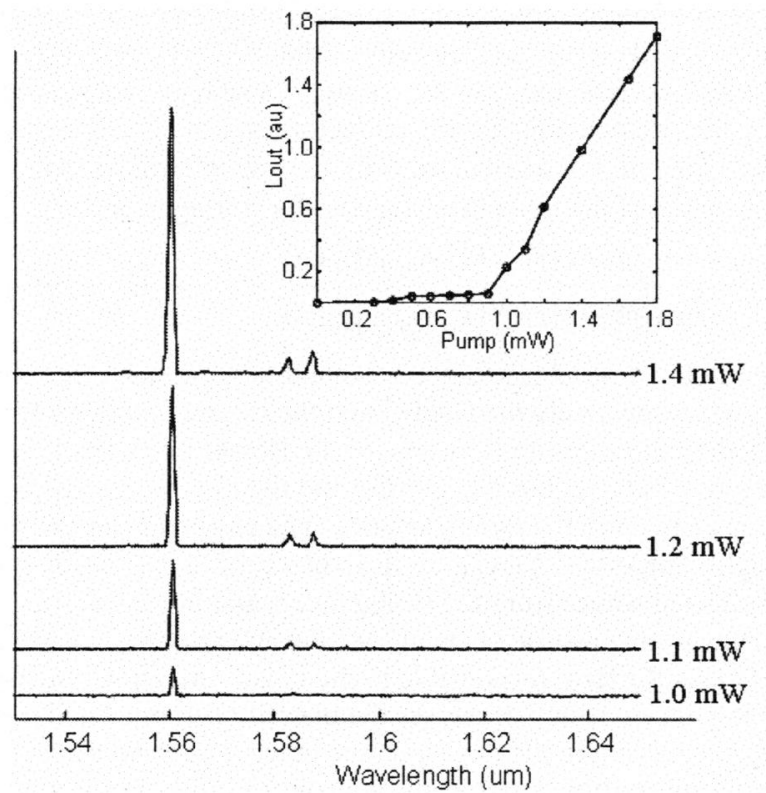

Fig. 2. The evolution of the emitted spectrum from the device shown in Fig. 1(a) as a function of the pump intensity. Inset - L-L curve.

Figure 2 depicts the emitted spectra from the $m=0$ laser for various pumping levels. The emitted light consists primarily of a single wavelength at $\lambda=1.56 \mu \mathrm{m}$, very close to the target wavelength of $1.55 \mu \mathrm{m}$ the 
resonator was designed for. The inset of Fig. 2 shows an L-L curve of the same device, indicating a threshold at $P_{t h}=900 \mu \mathrm{W}$. At high pump levels $\left(\sim 1.5 \mathrm{xP}_{\mathrm{th}}\right)$, additional two emission lines appear at longer wavelengths $(\sim 1.59 \mu \mathrm{m})$. We attribute these modes to emission from the external Bragg grating region [14].

Figure 3 shows the emitted spectra from the $m=4$ nano-laser for increasing pump levels. For pumping levels below $1 \mathrm{~mW}\left(\sim 2 \times \mathrm{P}_{\mathrm{th}}\right)$, the spectrum consists of a single emission line at $\lambda=1.58 \mu \mathrm{m}$. As the pump intensity exceeds that level, another mode appears at $\lambda=1.585 \mu \mathrm{m}$ (Fig. 3(a)). When the pump level is increased even further (Fig. 3(b)), the intensity of the new mode increases while the intensity of the originally lasing mode decreases, indicating mode switching. We attribute the blue-shifting of the lasing wavelength to generation of free carriers by the increasing pump which reduces the refractive index of the semiconductor membrane.
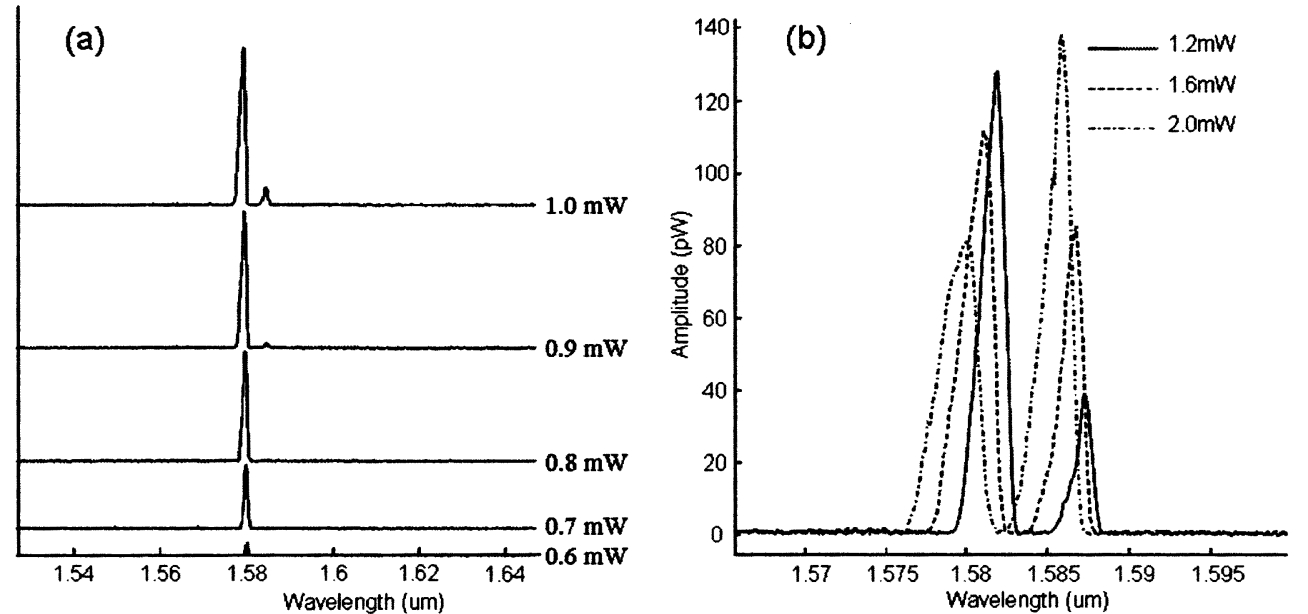

Fig. 3. The evolution of the emitted spectrum from the device shown in Fig. 1(b) as a function of the pump intensity: (a) Low pump levels and (b) zoom-in on the lasing wavelengths at high pump levels.

\section{Conclusions}

We demonstrated circular nano-lasers based on Bragg reflection, realized in a thin membrane of InGaAsP active semiconductor material. Single and multi mode lasing is observed from the devices, depending on the cavity dimensions. In the larger cavities, a mode-switching process is observed when the pump intensity is increased above twice the threshold level. Lasing was achieved at room temperature under pulsed optical pumping conditions at sub $\mathrm{mW}$ threshold levels. Such cavities can easily be integrated with other photonic devices such as photonic crystals waveguides and distributed feedback lasers to realize a compact, high functionality optical circuit.

\section{References}

[1] C. K. Madsen and J. H. Zhao, Optical Filter Design and Analysis: A Signal Processing Approach. New York: Wiley, 1999.

[2] B. E. Little, "Ultracompact Si-SiO2 microring resonator optical dropping filter", Opt. Lett. 23, 1570 (1998);

[3] A. Yariv, "Critical Coupling and Its Control in Optical Waveguide-Ring Resonator Systems", IEEE Photonics Technol. Lett. 14, 483 (2002).

[4] J. E. Heebner and R. W. Boyd, “'Slow' and 'fast' light in resonator-coupled waveguides", J. Mod. Opt. 49, 2629 (2002);

[5] C. Y. Chao and L. J. Guo, "Biochemical sensors based on polymer microrings with sharp asymmetrical resonance", Appl. Phys. Lett. 83, $1527(2003)$

[6] A. B. Matsko, L. Maleki, A. A. Savchenkov and V. S. Ilchenko, "Whispering gallery mode based optoelectronic microwave oscillator “, J. Mod. Opt. 50, 2523 (2003).

[7] See for example K.J. Vahala, "Optical microcavities", Nature 424, 839-846 (2003) and references therein.

[8] J. Scheuer and A. Yariv, "Coupled-Waves Approach to the Design and Analysis of Bragg and Photonic Crystal Annular Resonators", IEEE J. Quantum Electron. 39, p. 1555, 2003.

[9] C. Wu, M. Svilas, M. Fallahi, T. Makino, J. Glinski, C. Maritan and C. Blaauw, "Optically pumped surface-emitting DFB GaInAsP/InP lasers with circular grating", Electron. Lett. 27, p. 1819, 1991.

[10] D. Labilloy, H. Benisty, C. Weisbuch, T. F. Krauss, C. J. M. Smith, R. Houdre and U. Oesterle, "High-finesse disk microcavity based on a circular Bragg reflector", Appl. Phys. Lett. 73, p. 1314, 1998.

[11] J. Scheuer, W. M. J. Green, G. DeRose and A. Yariv, "Low Threshold Two-Dimensional Annular Bragg Lasers", Opt. Lett. 29, p. 2641, 2004.

[12] W. M. J. Green, J. Scheuer, G. DeRose and A. Yariv, "Vertically emitting annular Bragg lasers using polymer epitaxial transfer", Appl. Phys. Lett. 85, p. 3669, 2004.

[13] L. A. Coldren and S. W. Corzine, Diode Lasers and Photonic Integrated Circuits. New York: Wiley, 1995.

[14] J. Scheuer W. Green, G. DeRose and A. Yariv, “Annular Bragg Defect mode Resonators", Proceedings of SPIE -- Volume 5333 Laser Resonators and Beam Control VII, Alexis V. Kudryashov, Editor, p. 183, 2004. 